\title{
Los procesos de clasificación de usuarios y su impacto en la atención en las salas de urgencias a nivel hospitalario ${ }^{1}$
}

\author{
Consuelo Cubero Alpízar ${ }^{2}$
}

Kattia Medina Arias ${ }^{3}$

Institución: Universidad de Costa Rica

\section{COMO CITAR}

Cubero, C. y Medina, K. (abril, 2013). Los procesos de clasificación de usuarios y su impacto en la atención en las salas de urgencias a nivel hospitalario. Rev. Actual en Costa Rica, 24, 1-12. Recuperado de:: <http://www.revenf.ucr.ac.cr/triage.pdf> ISSN 1409-4568

\section{RESUMEN}

Este artículo es un avance del proyecto de investigación No.421-B1-237 titulado "Estrategias para la atención y clasificación de usuarios (as) en los servicios de urgencias a nivel hospitalario". Tiene como objetivo general valorar las estrategias para la atención de usuarios (as) en los servicios de urgencias hospitalarias. Se aplicó la metodología de Práctica de Enfermería Basada en la Evidencia (PEBE) la cual consta de cinco pasos: formulación de preguntas susceptibles de ser respondidas; búsqueda bibliográfica exhaustiva; lectura crítica de los documentos obtenidos; aplicación de la intervención más adecuada, y evaluación de la intervención. Dentro de los principales resultados se encuentra que las modalidades de clasificación de usuarios (as) son variadas desde las estructuradas que son fáciles de replicar hasta la atención aleatoria por llegada, las investigaciones señalan que esta atención estructurada se convierte en la herramienta fundamental para los y las profesionales de Enfermería, ya que se convierten junto con un entrenamiento en piezas fundamentales para la clasificación de usuarios (as) por estos profesionales. Se concluye que existe poca información asociada la aplicación de sistemas de clasificación y la mortalidad en los servicios de urgencias, por lo que se identifica un vacío de información en la temática que debe motivar a la realización de más y mejor investigación al respecto.

Palabras clave: Triage, clasificación, profesionales, servicios de urgencias, enfermería.

\footnotetext{
${ }^{1}$ Fecha de recepción: 12 de enero del 2012

Fecha de aceptación: 15 de marzo del 2013

${ }^{2}$ Enfermera, docente de la Escuela de Enfermería, Master en Gestión del Riesgo y Respuesta a Emergencias. Correo electrónico: ccuberoalpzar@gmail.com

${ }^{3}$ Enfermera, docente de la Escuela de Enfermería, Master en Nutrición. Correo electrónico: kattiamarcela@gmail.com
} 


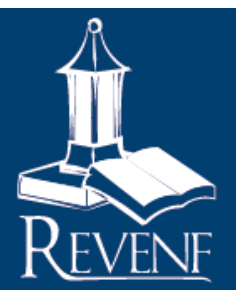

\section{The classification of users processes and their impact on care in emergency rooms in hospitals $^{1}$}

Institución: Universidad de Costa Rica

\section{CITED}

Cubero, C. y Medina, K. (abril, 2013). The classification of users processes and their impact on care in emergency rooms in hospitals. Rev. Actual en Costa Rica, 24, 1-12. Recovered: <http://www.revenf.ucr.ac.cr/triage.pdf> ISSN 1409-4568

Keywords: Triage, classification, professional, emergency services, nursing

\footnotetext{
${ }^{1}$ Date of receipt: January 12, 2013

Date of acceptance: March 15, 2013

${ }^{2}$ Enfermera, docente de la Escuela de Enfermería, Master en Gestión del Riesgo y Respuesta a Emergencias. E mail: ccuberoalpzar@gmail.com

${ }^{3}$ Enfermera, docente de la Escuela de Enfermería, Master en Nutrición. E mail: kattiamarcela@gmail.com
} 


\section{INTRODUCCIÓN}

Este artículo presenta un avance de resultados del proyecto No.421-B1-237 titulado "Estrategias para la atención y clasificación de usuarios (as) en los servicios de urgencias a nivel hospitalario" aprobado por la Vicerrectoría de Investigación de la Universidad de Costa Rica.

Los sistemas de clasificación de pacientes tienen cerca de 200 años de existir, su origen se ubica en las guerras napoleónicas, Illesca (2006) destaca que nacen cuando,

"El barón Dominique Jean Larrey, jefe médico de las tropas, recibe del emperador Napoleón Bonaparte una orden simple, pero controvertida: "los soldados que no tengan capacidad de recuperarse, deben dejarse en el frente de batalla, sólo deben de llevarse al hospital aquellos que puedan restablecerse"'(s.p).

Posteriormente, estos sistemas fueron evolucionando hasta ser acogidos por los servicios de urgencias hospitalarias y se realizaron adaptaciones de acuerdo con las características del lugar donde se aplicaron y a las necesidades identificadas.

Es así como el médico Larrey, aplicó por primera vez un sistema de clasificación de usuarios (as). Actualmente, participan en la aplicación de dicho método muchas personas entre ellas profesionales y no profesionales que lo realizan con base en el sentido común.

Diversos trabajos de investigación destacaron el problema de la saturación de los servicios de urgencias, como lo afirma Flores (2011),

La saturación de los servicios de urgencias hospitalarios (SUH) es un problema global que afecta a millones de pacientes cada día. Debe considerarse como un problema que afecta a la calidad y la seguridad de los pacientes, y no sólo como un asunto que afecte a la organización. (p.59)

Sempere, Peiró, Sendra, Martínez y López (1999), agregan que la saturación en los SUH es un aspecto de preocupación debido al aumento de su utilización, sobre todo porque se presentan casos que pueden ser atendidos en otros niveles del Sector Salud y que generan una distribución inequitativa de los recursos que se invierten en situaciones que no son urgencias, tanto en recurso humano, material como estructural. A esta misma conclusión llegan las investigaciones de Sanchez y Bueno (2005) y Marín (2008). Además Salmerón, Jiménez, Miró y Sánchez (2011) indicaron que “...hasta el $50 \%$ de los pacientes que consultan el SUH lo hacen por condiciones poco complejas que podrían ser resueltas en dispositivos extrahospitalarios”. (p. 346)

Esta panorámica no es ajena a la realidad costarricense, el desequilibrio entre la oferta y la demanda en la atención muchas veces pone en riesgo la seguridad de los usuarios (as) del sistema, por lo que genera crisis en la 
atención que se brinda a las personas debido entre otros factores al limitado acceso a los servicios de salud, la insatisfacción en la atención que se brinda en el primer nivel de atención. Además, se debe considerar que hay fácil acceso a los servicios de emergencias hospitalarios para personas aseguradas y no aseguradas, aspecto que incide en la saturación de estos servicios.

En ese mismo orden surgen estrategias para paliar el problema, todas centradas en la agilización de la atención de los usuarios (as) en los servicios. La más difundida es la implementación de sistemas de clasificación utilizados en los SUH para dar prioridad en la atención a aquellas con patologías que ponen en riesgo la vida.

En la actualidad, en los servicios de urgencias hospitalarios, existe gran cantidad de sistemas de clasificación de usuarios (as), que se adaptan al contexto institucional y que han surgido de procesos investigativos; no obstante, otros responden a la subjetividad del funcionario o profesional que tiene la potestad de recibir a una persona en esa área hospitalaria o que su actuar obedece a la necesidad de aminorar la cantidad de usuarios (as) de este tipo de servicio.

La mezcla entre los diferentes escenarios hospitalarios y las personas que han aplicado los sistemas de clasificación ha llevado al surgimiento de diferentes modelos para la clasificación, algunos muy sistemáticos y fáciles de reproducir en otros contextos, según Candela, Cámara, Valenzuela, Porcel, y Romero-Nieva (2006) los más destacados son "Australian Triage Scale (ATS), Canadian Triage and Acuity Scale(CTAS), Manchester Triage Scale (MTS), Emergency Severity Index (ESI), Modelo andorrano de Triage(MAT), Sistema Español de Triage (SET)"(s.p.)

De la misma manera existen otros sistemas para la priorización de la atención en los servicios de urgencias hospitalarios menos estandarizados con criterios más subjetivos en su aplicación, en este sentido Illesca (2006) menciona el índice de triage, índice de trauma, sistema cronológico, sistema de lotería, sistema por criterio, sistema de puntuación de la severidad de las lesiones, sistema de servicio de espera, que al igual que los sistemas antes mencionados tratan de clasificar la condición de cada persona que recurre a este tipo de servicios para mejorar su atención en lo referente a efectividad y calidad.

El objetivo que se planteó en este estudio fue valorar si hay reducción en la mortalidad y los tiempos de espera con la aplicación de un sistema estructurado de clasificación de usuarios (as) en los servicios de urgencias hospitalarias en comparación con la atención aleatoria por llegada.

Esta investigación tiene implicaciones muy importantes para la labor que desempeña el o la enfermera, debido a que proporciona elementos científicos para la toma de decisiones que contribuyan al mejoramiento de la atención y por consiguiente mayor seguridad para los y las usuarias de los servicios de urgencias hospitalarios. 


\section{MATERIALES Y MÉTODOS}

Esta investigación se basó en la metodología de la Enfermería Basada en la Evidencia (EBE) la cual es definida como “...un enfoque de toma de decisiones en el cual los clínicos usan la mejor evidencia disponible, en consulta con los pacientes, para decidir cuál es la opción que es más aceptable con lo mejor que tenga el paciente.” (Urra, Retamal, Tapia y Rodríguez, 2010, p.2)

Se aplicaron cinco pasos que son: formulación de la pregunta clínica en formato PICO, búsqueda de la información científica, lectura crítica de la información científica recopilada, implementación de la evidencia científica, evaluación de la implementación.

Respecto de la pregunta clínica las investigadoras se plantearon la siguiente: ¿En personas que asisten a los servicios de urgencias, la aplicación de un sistema estructurado de clasificación en comparación con la atención aleatoria por llegada, reduce la mortalidad y los tiempos de espera?

Como segundo paso en esta metodología de investigación basada en evidencia, se llevó a cabo una revisión bibliográfica de artículos científicos; esto con el fin de recuperar la mayor cantidad de documentos científicos que trataran la temática planteada de acuerdo con el tipo de pregunta. Las bases de datos utilizadas fueron Medline Plus y LILACS, GOOGLE ACADEMICO y EBSCO. Los descriptores introducidos en las bases fueron: ("triage"[MeSH Terms] OR "triage"[All Fields]) AND effectiveness[All Fields] AND ("mortality"[Subheading] OR "mortality"[All Fields] OR "mortality"[MeSH Terms]) “Triage”, "clasificación and urgencias", "efectividad triage", "servicio de urgencia hospitalario y "personal que aplica el sistema de clasificación", "mortalidad and clasificación and servicios urgencias" tanto en idioma inglés como en español con el fin de obtener la mayor cantidad de resultados. En una primera búsqueda se encontraron 5000 documentos, luego se aplicaron límites en la búsqueda cómo fue el tener formato de artículo científico, texto completo y el año de publicación (1998 - 2012) y fueron identificados 100 artículos, los cuales serán sometidos al análisis crítico en una fase posterior.

Se destaca que el presente documento corresponde a un avance de los resultados encontrados hasta el momento sobre la temática planteada, ya que la investigación se encuentra en el análisis crítico de la información recolectada.

\section{Consideraciones éticas}

Para cada material que se utilizó, en el desarrollo de la presente investigación, se respetaron los derechos de autor, incorporando a lo largo del texto las citas bibliográficas correspondientes. Respecto del tratamiento de los documentos recolectados (datos) el manejo que se hace de los mismos concuerda con la metodología de práctica de enfermería basada en la evidencia. 


\section{RESULTADOS}

De 100 artículos recuperados, se han revisado 20 hasta el momento, de los cuales sólo cuatro guardan una calidad de "buena". Estos se presentan a continuación:

\begin{tabular}{|c|c|c|c|}
\hline Título & $\begin{array}{c}\text { F. } \\
\text { Publi } \\
\text { c. }\end{array}$ & Calidad & Criterio \\
\hline $\begin{array}{l}\text { Estudio de Triage y } \\
\text { Tiempos de Espera en } \\
\text { un servicio de } \\
\text { urgencias hospitalario }\end{array}$ & 1998 & Buena & $\begin{array}{l}\text { El artículo presenta una metodología clara, la información y } \\
\text { tema de investigación concuerdan con la pregunta planteada en } \\
\text { la investigación. De la información importante se encuentra que } \\
\text { "los tiempos de espera se vienen utilizando como medida de } \\
\text { control de calidad y en ellos influyen recursos humanos, } \\
\text { materiales y organizativos del propio servicio" (p.101). Además } \\
\text { afirma que "el Triage se consolida como un sistema eficaz de } \\
\text { ordenación del trabajo asistencial, para dar respuesta inmediata } \\
\text { a la demanda masificada dentro de una S.U.H., facilitando el } \\
\text { trabajo del personal y disminuyendo la ansiedad de los } \\
\text { enfermos y familiares ante la espera" (p.100). El estudio } \\
\text { concluye que 1. La sobrecarga de trabajo condiciona } \\
\text { irremediablemente una disminución en la calidad asistencial, y } \\
\text { para esto existen dos soluciones: 1. }{ }^{a} \text { ) disminuir la demanda } \\
\text { mediante la mejora de la asistencia prehospitalaria y la } \\
\text { educación sanitaria de la población, y 2. }{ }^{a} \text { ) aumentar las } \\
\text { prestaciones, organizando el trabajo y creando circuitos de } \\
\text { triage. } 2 \text {. El triage se consolida como un sistema eficaz de } \\
\text { ordenación del trabajo asistencial, para dar respuesta inmediata } \\
\text { a la demanda masificada dentro de los S.U.H., facilitando el } \\
\text { trabajo del personal y disminuyendo la ansiedad de los } \\
\text { enfermos y familiares ante la espera. 3. Los grupos de } \\
\text { clasificación establecidos (códigos de color) muestran una } \\
\text { elevada relación con el destino del paciente (eficacia del triage), } \\
\text { si bien existe una tendencia a la sobrevaloración de las } \\
\text { patologías graves en nuestros resultados. 4. El estudio de } \\
\text { tiempos de espera nos revela que, para una proporción de } \\
\text { urgencias importante, la oferta asistencial sigue siendo } \\
\text { deficitaria respecto a la demanda, lo que obligará a estudios } \\
\text { posteriores. }\end{array}$ \\
\hline $\begin{array}{lr}\text { Triage en } & \text { urgencias: } \\
\text { Facultativo } & \text { versus } \\
\text { Enfermería } & \end{array}$ & 2003 & Buena & $\begin{array}{l}\text { Tiene una metodología rigurosa, es acorde con el objetivo } \\
\text { planteado. Dentro de la información relevante se encuentra: " } \\
\text { en nuestro trabajo comparamos la eficacia del triage realizado } \\
\text { por Facultativos o Enfermería, llegando a la conclusión que no } \\
\text { es estadísticamente diferente."(p.150) Conclusiones: La } \\
\text { realización del triage del personal de enfermería no es } \\
\text { estadísticamente diferente con el realizado por personal } \\
\text { facultativo. La mayoría de los pacientes que acuden a } \\
\text { Urgencias Hospitalarias lo hacen por iniciativa propia, suelen }\end{array}$ \\
\hline
\end{tabular}




\section{Revista Electrónica Enfermeria Actual en costa Rica}

\begin{tabular}{|c|c|c|c|}
\hline & & & $\begin{array}{l}\text { presentar una patología banal (Prioridad 3; Urgencia no } \\
\text { hospitalaria) y tras finalizar su asistencia la mitad de los } \\
\text { pacientes son dados de alta con el problema solucionado. Todo } \\
\text { esto nos obliga a pensar en la efectividad de un sistema de } \\
\text { priorización y una consulta especial donde atender esta } \\
\text { prioridad } 3 \text { para no sobrecargar el resto de consulta }\end{array}$ \\
\hline $\begin{array}{l}\text { Patient expectations of } \\
\text { emergency department } \\
\text { care: phase II - a cross- } \\
\text { sectional survey }\end{array}$ & 2006 & Buena & $\begin{array}{l}\text { Mencionan como el sistema de triage canadiense a impactado } \\
\text { en los tiempos de espera. La conclusión del estudio es que las } \\
\text { expectativas del usuario están centradas en mejorar los tiempos } \\
\text { de espera en el servicio. Además una mayor atención a los } \\
\text { tiempos de espera y la comunicación personal puede aumentar } \\
\text { tanto la eficiencia la satisfacción del paciente }\end{array}$ \\
\hline $\begin{array}{l}\text { Gestión de la demanda } \\
\text { de urgencias: ¿Quiero } \\
\text { que el médico me vea } \\
\text { ahora! ¿Cómo lo } \\
\text { hacemos? }\end{array}$ & 2010 & Buena & $\begin{array}{l}\text { Tiene metodología rigurosa uno de los principales resultados } \\
\text { para responder a la pregunta planteada es el siguiente: "La } \\
\text { consulta de enfermería de triage evita en un } 41 \% \text { las visitas } \\
\text { médicas excedentes en ese día." } 564 \\
\text { La conclusión de la investigación es la siguiente: Valoración } \\
\text { elevada por parte de todo el personal de la consulta de triaje. } \\
\text { Las mejoras y la disminución de carga de trabajo lo refieren } \\
\text { principalmente administrativos y médicos. }\end{array}$ \\
\hline
\end{tabular}

\section{DISCUSIÓN}

Los servicios de urgencias están experimentando serios problemas por la saturación que padecen producto -muchas veces- del uso indiscriminado que se hace de ellos por parte de los usuarios (as).

Un aspecto que está repercutiendo en esta saturación es la diferencia conceptual entre la urgencia y la emergencia; la primera es una percepción del individuo (subjetiva) quien experimenta esa necesidad de ser atendido con rapidez, mientras que la emergencia tiene un componente más técnico, más objetivo ya que se trata de una condición que pone en riesgo la vida de la persona que la padece.(Artiguez, 2007; Zaragoza et al, 2009; Carbonell, Mira, Aranaz y Pérez, 2004; Gómez, 2006).

De esta manera muchas personas acuden a estos servicios por problemas de poca relevancia que pudieran ser resueltos en otro nivel de atención, ocasionando aumento en los tiempos de espera y saturación en el servicio.

Investigaciones como la de Irola et al. (2004) enfatizan la importancia de la calidad en la atención en función de la resolución del problema, las condiciones ambientales y la disminución en los tiempos de espera pero 
no relacionados con un sistema de clasificación óptimo, si no mediados por otros aspectos como el trato recibido o las condiciones estructurales de las salas de espera y tratamiento.

La calidad en los servicios de urgencia según la investigación de SEMES-Insalud (2001) es medida por una serie de indicadores dentro de los que se encuentran los tiempos de espera, la mortalidad, la tasa de retorno de los usuarios (as) al servicio, tiempo de permanencia en el servicio, proporción de ingresos, codificación del diagnóstico de alta entre otros. El análisis de estos indicadores me indica la calidad de la atención brindada al usuario (a) en el servicio.

Debido a que muchos de los indicadores antes mencionados tienen una estrecha relación con el sistema de clasificación y el personal que los aplica, es que resultan interesantes los resultados que se han obtenido hasta el momento en esta investigación.

El principal dato es que, a pesar de que los sistemas de triage provienen de un medio extrahospitalario adaptados con el paso de los años a las salas de urgencias hospitalarias y a las características propias del país y la cultura donde se desarrollan, estos son efectivos. Esto se evidencia por la agilización de la atención en las salas de urgencias. (Álvarez, Gorostidi, Rodríguez, Antuña, y Alonso, 1998).

Situación que es ratificada en la investigación de Cooke, Watt, Wertzle, \& Quan, (2006) donde afirma la efectividad del sistema de Triage Canadiense. Además, agrega el hecho de que las y los pacientes cuando asisten a las salas de urgencias tienen la expectativa de una atención rápida y una buena comunicación con el personal; éstos como criterios de calidad en la atención.

Otro aspecto relevante de lo analizado hasta el momento se relaciona con el personal que realiza el triage donde se evidencia que no hay diferencia en efectividad del triage aplicado por el profesional de Enfermería y el facultativo ${ }^{1}$ (Parrilla, 2003); más aún en la investigación de Rafols, et al. (2010) se afirma que se da una disminución en la carga de trabajo aplicando triage por Enfermería, en palabras de los autores dicen que "La consulta de enfermería de triage evita en un $41 \%$ las visitas médicas excedentes en ese día." (p.564)

Reafirmando lo anterior, la literatura resalta el papel del profesional de enfermería en este proceso, lo cual se encuentra sustentado por Soler, et al.(2010) mencionando que,

Dado que el triage no se fundamenta en diagnósticos y la concordancia interobservador hallada en las diferentes escalas ha resultado muy satisfactoria, el triage es reivindicado desde sus inicios por enfermería, que consigue muy buenos resultados incluso con escalas de 4 niveles de prioridad. Hay, sin embargo, sistemas que defienden que debe ser un médico experimentado de urgencias el encargado de realizarlo (sistema donostiarra de triage). (s.p.)

\footnotetext{
${ }^{1}$ De acuerdo al diccionario de la real academia Española el término se refiere a los médicos o relacionado con ellos
} 


\section{Revista Electrónica Enfermeria Actual en costa Rica}

En la misma línea Small (2010) señala que:

Es necesaria la formación especializada de los y las profesionales de enfermería en el área de emergencias ya que mejora los tiempos de espera, la satisfacción de los usuarios, reduce los problemas legales, entre muchas otras ventajas de la práctica de enfermería avanzada (especialización de enfermeras para los servicios de urgencias) (s.p.)

Además una revisión sistemática de Rowe et al.(2011) titulada "El papel de la enfermera de triage en ordenar o mitigar el hacinamiento en las salas de emergencias: una revisión sistemática" hace énfasis en que existe una gran cantidad de investigaciones al respecto sin embargo pocas tienen la calidad metodológica adecuada ya que se dejan de lado las circunstancias particulares que mediaron en las intervenciones. A pesar de estas limitantes sí existe evidencia de una disminución en el hacinamiento y los tiempos de espera que va de 20 a 50 minutos dependiendo del estudio.

Por su parte Robinson (2012) presenta como resultados más importantes de su estudio

Principales conclusiones extraídas de las evaluaciones incluyeron que una disminución en la estancia hospitalaria fue relacionada con el uso del protocolo y las enfermeras fueron capaces de iniciar las pruebas diagnósticas y los tratamientos adecuadamente. Estas conclusiones se aplican a niveles de gravedad 3 y 4 , los cuales requieren de pruebas ya sea poco o nada o que requieren de pruebas para facilitar la decisión de disposición. (s.p.)

De esta manera es claro como los sistemas de triage se han convertido en una herramienta que le brinda calidad y agilidad a los servicios de urgencias hospitalarias, aspecto que disminuye la saturación y los tiempos de espera. Sin embargo, es importante destacar que existe poca información con respecto a la mortalidad en estos centros, así como la asociación de esta variable con dichos sistemas de clasificación y el personal que los aplica.

\section{CONCLUSIONES}

La información recabada hasta el momento señala como los y las profesionales de enfermería son pieza fundamental para agilizar la atención en los servicios de urgencias y para la aplicación de los sistemas de Triage. Existe poca información asociada la aplicación de sistemas de clasificación y la mortalidad en los servicios de urgencias, por lo que se identifica un vacío de información en la temática que debe motivar a la realización de más y mejor investigación al respecto. 


\section{Bibliográfica}

Álvarez, B., Gorostidi J., O. Rodríguez, O., Antuña, A. y . Alonso P (1998). Estudio de Triage y Tiempos de Espera en un servicio de urgencias hospitalario. Emergencias, 10(2), 100-104

Artiguez, R.(2007). Diferencias entre Urgencias y Emergencias. Recuperado de: http://www.semescyl.org/?q=node/128

Candela, M., Cámara, S., Valenzuela, A., Porcel, A. y Romero-Nieva, J.(2006). Recepción, acogida y clasificación de pacientes en urgencias RAC \& TRIAGE.

Carbonell, A. M., Torregrosa, Mira J. J., Aranaz, J., Pérez, V. (2004). Evaluación de la calidad de la asistencia en el servicio de urgencias hospitalario a través de la revisión de informes clínicos. Emergencias, 16, 137-142.

Cooke, T., Watt, D., Wertzler, W. \& Quan, H.( 2006) Patient expectations of emergency department care: phase II--a cross-sectional survey. CJEM, 8(3), 148-157

Flores, C. (2011). La saturación de los servicios de urgencias: una llamada a la unidad. Emergencias, 23, (1), 5964

Gómez, J. (2003). Clasificación de pacientes en los servicios de urgencias y emergencias: Hacia un modelo de triaje estructurado de urgencias y emergencias. Emergencias, 15,165-174.

Gómez, J.(2006). Urgencia, gravedad y complejidad: un constructo teórico de la urgencia basado en el triaje estructurado. Emergencias, 18, 156-164

Illesca, G. (2006). Triage: atención y selección de pacientes. TRAUMA, 9(2), 48-56.

Iraola, M., Fernández, D., Liriano, J., Rodríguez, G., Rodríguez, A. y Rojas, O. (2004). Satisfacción de los pacientes atendidos en urgencias y posteriormente hospitalizados en un hospital universitario cubano. Emergencias, 16, 252-257.

Marín, J. (2008) Causas y Efectos de la Saturación del Servicio de Emergencias de la Clínica de Chomes, Puntarenas.

http://biblioteca.icap.ac.cr/BLIVI/TESIS/2008/Marin_Gomez Jose Pablo 2008 SA.pdf

Parrilla, F., Cárdenas, D., Vargas, D., Parrilla, E., Díaz, M. y Cárdenas, A. (2003). Triage en urgencias: Facultativo versus Enfermería. Emergencias, 15, 148-151.

Rafols, A., Sieira, M.A., De Ciurana, M., Franco, P.Font, P. y Torrent, S(2010 ). Gestión de la demanda de urgencias: ¡Quiero que el médico me vea ahora! ¿Cómo lo hacemos? Elsevier España,S.L. y SEMERGEN doi:10.1016/j.semerg.2010.04.012 
Robinson, D.J. (2012). An Integrative Review: Triage Protocols and the Effect on ED Length of Stay. $J$ Emerg Nurs, Recuperado de: http://www.ncbi.nlm.nih.gov/pubmed/22513188

Rowe, B.H., Villa-Roel, C., Guo, X., Bullard, M.J., Ospina, M., Vandermeer, B., Innes, G., Schull, M.J. \& Holroyd, B.R. (2011). The role of triage nurse ordering on mitigating overcrowding in emergency departments: a systematic review. Acad Emerg Med. 18(12), 1349-57. doi: 10.1111/j.1553-2712.2011.01081.x.

Rowe, B.H., Guo, X., Villa-Roel, C., Schull, M., Holroyd, B., Bullard, M., Vandermeer, B., Ospina, M. \&Innes $\mathrm{G}$. The role of triage liaison physicians on mitigating overcrowding in emergency departments: a systematic review. Acad Emerg Med. 18(2), 111-20. doi: 10.1111/j.1553-2712.2010.00984.x

Salmerón, J., Jiménez, L., Miró, O., Sánchez, M. (2011). Análisis de la efectividad y seguridad de la derivación sin visita médica desde el triaje del Servicio de Urgencias Hospitalario por personal de enfermería acreditado utilizando el Programa de Ayuda al Triaje del Sistema Español de Triaje. Emergencias, 23, 346-355.

Sánchez, J. y Bueno, A. (2005) Factores asociados al uso inadecuado de un servicio de urgencias hospitalario. Emergencias 17, 138-144.

SEMES-Insalud (2001). Calidad en los servicios de urgencias.Indicadores de calidad. Emergencias. 13 Recuperado de: http://www.semes.org/revista/vol13_1/60-65.pdf

Sempere, T., Peiró, S., Sendra, P., Martinez, C., López, I. (1999).Validez del protocolo de adecuación de urgencias hospitalarias. Revista Española de Salud Pública, 73, 465-479.

Small, V.(2010). El desarrollo de un rol de practica avanzada en enfermería de urgencias y emergencias: reflexiones desde la experiencia de Irlanda. Emergencias, 22, 220-225

Soler, W., Gómez, M., Bragulat, E. y Álvarez, A.(2010). El triaje: herramienta fundamental en urgencias y emergencias. An. Sist. Sanit. Navar., 33 (Supl. 1), 55-68

Zaragoza, M., Calvo, C., Saad, T., Morán, F.J., Pizarro, S. y Hernández, P.(2009). Evolución de la frecuentación en un servicio de urgencias hospitalario. Emergencias, 21 339-345

Urra, E., Retamal, C., Tapia, C. y Rodríguez, M. (2010). Enfermería basada en la evidencia: qué es, sus características y dilemas. Invest. educ. enferm 28 (1) Recuperado de: http://www.scielo.org.co/scielo.php?pid=S0120-53072010000100013\&script $=$ sci arttext 